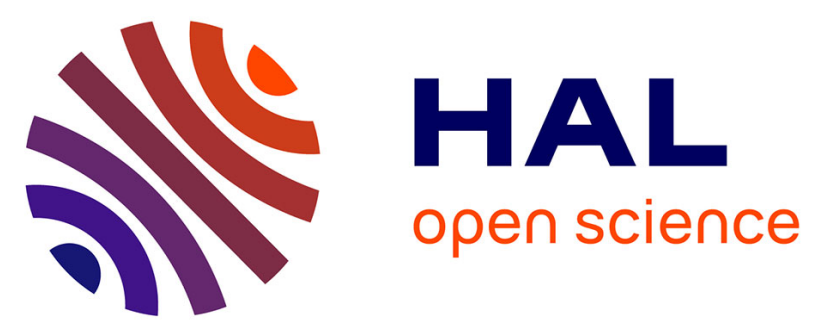

\title{
Vericiguat for the treatment of heart failure: mechanism of action and pharmacological properties compared with other emerging therapeutic options
}

\author{
Jean-Sébastien Hulot, Jean-Noël Trochu, Erwan Donal, Michel Galinier, \\ Damien Logeart, Pascal de Groote, Yves Juilliere
}

\section{To cite this version:}

Jean-Sébastien Hulot, Jean-Noël Trochu, Erwan Donal, Michel Galinier, Damien Logeart, et al.. Vericiguat for the treatment of heart failure: mechanism of action and pharmacological properties compared with other emerging therapeutic options. Expert Opinion on Pharmacotherapy, 2021, 22 (14), pp.1847-1855. 10.1080/14656566.2021.1937121 . hal-03280184

\section{HAL Id: hal-03280184 \\ https://hal.science/hal-03280184}

Submitted on 12 Jul 2021

HAL is a multi-disciplinary open access archive for the deposit and dissemination of scientific research documents, whether they are published or not. The documents may come from teaching and research institutions in France or abroad, or from public or private research centers.
L'archive ouverte pluridisciplinaire HAL, est destinée au dépôt et à la diffusion de documents scientifiques de niveau recherche, publiés ou non, émanant des établissements d'enseignement et de recherche français ou étrangers, des laboratoires publics ou privés. 


\section{Vericiguat for the treatment of heart failure: mechanism of action and pharmacological properties compared with other emerging therapeutic options}

Jean-Sébastien Hulot, $M D^{*}, 1,2$; Jean-Noël Trochu, $M D^{3}$; Erwan Donal, $M D, \mathrm{PhD}^{4}$; Michel Galinier, $M D^{5}$; Damien Logeart, $M D^{6}$; Pascal de Groote, $M D^{7}$; Yves Juillière, $M D^{8}$

1 Université de Paris, INSERM, PARCC, F-75006 Paris, France ;

2 CIC1418 and DMU CARTE, AP-HP, Hôpital Européen Georges-Pompidou, F-75015, Paris, France; Email: jean-sebastien.hulot@aphp.fr

3 Université de Nantes, CHU Nantes, CNRS, INSERM, l'institut du Thorax, F-44000 Nantes, France.

Email: jeannoel.trochu@chu-nantes.fr

4 LTSI INSERM U1099, Service de Cardiologie et Maladies Vasculaires, Centre Hospitalier Universitaire de Rennes, Université Rennes 135000 Rennes, France. Email: erwan.donal@chu-rennes.fr

5 Department of Cardiology, Toulouse-Rangueil University Hospital, Toulouse University School of Medicine, TSA 50032, 31059 Toulouse Cedex 9, France. Email: galinier.m@ chu-toulouse.fr

6 Department of Cardiology, Lariboisière Hospital, Paris, France; Université de Paris, Paris, France.

Email: damien.logeart@aphp.fr

7 CHU Lille, Service de Cardiologie, F-59000 Lille, France, INSERM U1167, Institut Pasteur de Lille, F-59000 Lille, France. Email: pascal.degroote@chru-lille.fr

8 Centre Hospitalier Universitaire de Nancy Brabois, 54500 Vandoeuvre Lès Nancy, France. Email: yves.juilliere@wanadoo.fr

*Author for correspondence: Jean-Sébastien Hulot, Hôpital Européen Georges Pompidou, 20 Rue Leblanc, F-75015, Paris, France. Email: jean-sebastien.hulot@aphp.fr 


\section{Abstract}

Introduction: The significant morbidity and mortality in patients with heart failure (HF), notably in the most advanced forms of the disease, justify the need for novel therapeutic options. In the last year, the soluble guanylate cyclase (sGC) stimulator, vericiguat, has drawn the attention of the medical community following the report of reduced clinical outcomes in patients with worsening chronic HF (WCHF).

Areas Covered: The authors review the available data on the mechanism of action of vericiguat (cyclic guanosine monophosphate (cGMP) pathway), its clinical development program, its role in HF management, and its future positioning in the therapeutic recommendations.

Expert opinion: CGMP deficiency has deleterious effects on the heart and contributes to the progression of HF. Different molecules, including nitric oxide (NO) donors, phosphodiesterase inhibitors, and natriuretic peptides analogues, target the NO-sCGcGMP pathway but have yielded conflicting results in HF patients. Vericiguat acts as a sGC stimulator thus targeting the NO-sGC-cGMP pathway by a different mechanism that complements the current pharmacotherapy for HF. Vericiguat has shown an additional statistical add-on therapy efficacy by reducing morbi-mortality in patients with WCHF. A better evaluation of HF severity might be an important determinant to guide the use of vericiguat among the available therapies.

Keywords (5-8): cGMP pathway; heart failure (HF); heart failure hospitalization (HFH); heart failure with reduced ejection fraction (HFrEF); reduced left ventricular ejection fraction (LVEF); soluble guanylate cyclase (sGC); SGC stimulator; ventricular remodeling; vericiguat; worsening chronic heart failure (WCHF).

\section{Article Highlights}

- cGMP deficiency is common in HF.

- Different therapies targeting the NO-sGC-cGMP pathway have been evaluated in HF patients but with conflicting results.

- Vericiguat is an SGC stimulator with an original mechanism of action.

- Vericiguat enhances SGC sensitivity to endogenous NO, thus exerts a more physiological action.

- Vericiguat reduces clinical outcomes in patients with worsening chronic HFrEF. 
- At a pharmacological level, vericiguat and ARNIs could have synergistic effects. 


\section{Introduction}

The economic burden of heart failure (HF) is increasing [1] with more than 26 million people worldwide affected by this condition [2]. After initial diagnosis and therapeutic management, HF patients can present a progressive deterioration of their HF symptoms leading to increased medical needs, intensification of therapies and hospitalization requirements $[3,4]$. These patients were recently defined as having worsening chronic heart failure (WCHF), and impose a growing healthcare burden because of recurrent medical needs and re-hospitalizations. In addition, quality of life is greatly impaired in patients with HF [5], and to a similar extent in patients with $\mathrm{HF}$ with reduced ejection fraction (HFrEF) and $\mathrm{HF}$ with preserved ejection fraction (HFpEF) [6].

Available pharmacological therapies in HFrEF consist of renin-angiotensinaldosterone system (RAS) blockers (either angiotensin-converting-enzyme inhibitor $[A C E I]$ or angiotensin receptor blockers [ARB]), or angiotensin receptor-neprilysin inhibitors (ARNls, such as sacubitril/valsartan) and mineralocorticoid receptor antagonists (MRAs), that are combined to beta-blockers and diuretics including loop diuretics [7]. When required, a cardiac resynchronization therapy \pm implantable cardioverter defibrillator are used. While these therapies address the main pathophysiological mechanisms activated in HF, the WCHF population remains at risk of high event rates despite their effectiveness. Consequently, there is a clinical unmet need to provide more therapeutic options investigating new pathways for patients with WCHF.

Among molecules, the impairment and deficiency of soluble guanylate cyclase (sGC) in cyclic guanosine monophosphate (CGMP) in $\mathrm{HF}$ is not directly targeted by available HF therapies. Deficiency of CGMP and resistance to the CGMP-dependent tissue effects of natriuretic peptides (NP) have however been reported for a long time in HF. Several drugs modulating the nitric oxide (NO)-cGMP pathways have been evaluated in HF, notably with NO donors (i.e., nitrates) or by reducing cGMP degradation with phosphodiesterase (PDE) inhibitors. More recently, novel drugs have been developed to directly increase the production of CGMP. In this context, novel classes of drugs called SGC stimulators (riociguat and vericiguat) and SGC activators (cinaciguat and ataciguat) were developed as an emerging therapy with a mechanism distinct from other available HF therapies [8], and vericiguat was particularly evaluated in HF patients. 


\section{Approved Use}

This paper will focus on vericiguat which has been developed and recently tested for the treatment of WCHF in adults with left ventricular ejection fraction (LVEF) below $45 \%$ to reduce the risk of cardiovascular (CV) death and heart failure hospitalization $(\mathrm{HFH})$, in combination with other HF therapies.

\subsection{Mechanism of Action of Vericiguat}

SGC has a key role in generating CGMP [9] with sGC-derived production of CGMP being essential for normal cardiac and vascular function [10-12]. cGMP is indeed a key second messenger to mediate vaso- and cardiac relaxation. In HF, NO availability and functionality of $\mathrm{sGC}$ are impaired, resulting in an increased oxidative stress and loss of cGMP production. In other terms, the NO-sGC-cGMP pathway is impaired in HF which may contribute to the progression of cardiovascular disease (CVD) in terms of endothelial, myocardial and vascular dysfunction [11,13]. Notably, a deficiency in cGMP is common to both HFrEF and HFpEF [12]. Oxidative stress can also stimulate autophagy, apoptosis, or necrosis, leading to the loss of cardiomyocytes and their replacement with collagen, and to fibrosis [14,15]. Moreover, left-ventricular remodeling/stiffness is associated with hypophosphorylation of titin found in cardiomyocytes which is associated with an impaired cGMP-dependent protein kinase G (PKG) activity [16-18]. Titin is the third myofilament of the cardiac muscle [19]. By having direct and indirect links with several signaling molecules and having multiple phosphorylation sites in the Z-disk, M-band, and I-band (containing the N2B element) segments, titin is recognized as a major regulator of cardiomyocyte stiffness. For example, the level of phosphorylation of the N2B element, notably through PKG, regulates the myofibrillar stiffness [19].

Vericiguat is an oral drug with a once-daily dosing schedule, that acts as a direct stimulator of the SGC enzyme. Vericiguat was developed to be indicated upon regulatory approval as a first-in-class therapy for WCHF [20]. Vericiguat has an optimized pharmacokinetic profile with a higher half-life as compared with the first sGC stimulator, riociguat, thus allowing the development of a once-daily oral administration regimen [21]. Vericiguat works in synergy with endogenous NO [13,22] 
which is considered as a nitrovasodilator to produce cGMP under low-NO conditions. Accordingly, vericiguat treatment is expected to restore the impaired NO-sGC-cGMP pathway leading to a diversity of pharmacological effects including improvements in cardiac and vascular functions, and reduction in pro-fibrotic and inflammatory pathways $[11,20]$ (Figure 1). By increasing cGMP, vericiguat is also estimated to induce vasorelaxation and improve regulation of vascular tone and myocardial dysfunction $[13,23,24]$. It would also attenuate left-ventricular remodeling by inducing PKGmediated phosphorylation of titin following the activation of PKG by CGMP [24]. On the hemodynamic level and based on the CHEST and PATENT studies on riociguat $[26,27]$, vericiguat is believed to improve the cardiac index through its vasodilator effect without exhaustion of this effect in contrast with other vasodilators. As for the extracardiac effects of vericiguat, this molecule has antiagregant and antifibrotic (potential benefit in kidney failure) properties [28]. Thus, vericiguat is anticipated to improve myocardial and endothelial functions in patients with HF and potentially with WCHF [9].

Importantly, intracellular CGMP is produced by two independent pathways, the NOsGC-cGMP and the NP-particulate guanylate cyclase (pGC)-cGMP pathway (Figure 2). While the NO-sGC-cGMP pathway is driven by sGC, the NP-pGC-cGMP pathway is driven by a membrane-bound, pGC. ARNIs such as sacubitril/valsartan are currently available for the treatment of HF, targeting the NP-pGC-cGMP pathway by inhibiting degradation of NP and many other vasoactive mediators. Thus, both vericiguat and sacubitril/valsartan can lead to CGMP augmentation through different pathways with a potentially synergistic effect between both agents, due to the contemporary stimulation of the two CGMP-generating pathways. It is also possible that vericiguat, sacubitril/valsartan, and other therapies that increase cGMP levels could lead to an increase of distinct and specific intracellular pools of cGMP which could then act synergistically. In this respect, a subgroup analysis of patients treated with sacubitril/valsartan in the Phase 3 VICTORIA trial suggested a potential synergistic effect of the vericiguat and sacubitril/valsartan combination [29].

On a different note, the $\mathrm{SGC}$ stimulator, vericiguat, differs from current therapies targeting the NO-sGC-cGMP pathway such as NO donors, PDE5 inhibitors, ACEls/ARBs, and ARNIs (sacubitril/valsartan), with no proven efficacy in HF for some of them (NO donors and PDE5 inhibitors): 
- While NO donors act upstream of the NO-sGC-cGMP pathway, vericiguat restores cGMP production even under low-NO conditions and high oxidative stress.

- Unlike PDE5 inhibitors that act downstream of the NO-sGC-cGMP pathway by preventing the breakdown of CGMP, vericiguat directly stimulates SGC to increase cGMP concentration [8].

- Independent of RAS blockers (i.e. ACEls/ARBs and ARNIs) and sympathetic nervous system blockers (i.e. beta-blockers), SGC stimulation is a highly effective way to actively restore the NO-cGMP pathway.

- Although ARNIs increase CGMP levels by indirectly blocking neprilysin-mediated degradation of NP, vericiguat directly stimulates SGC to significantly increase intracellular cGMP concentration [6,40,41].

Lastly, vericiguat has been shown to have advantages over SGC activators such as cinaciguat. Cinaciguat was administered as an intravenous infusion in patients with acute decompensated HF but induced hypotension without improving dyspnea or the cardiac index in the COMPOSE trials [42] In contrast to vericiguat, cinaciguat activates sGC irrespective of NO bioavailability, which may support higher vasodilatation effects [43]. Cinaciguat was consequently not further developed for HF patients.

\subsection{Clinical Development of Vericiguat in HFrEF}

SOCRATES-REDUCED (or Soluble Guanylate Cyclase Stimulator in Heart Failure with Reduced Ejection Fraction Study) was a Phase $2 b$ dose-finding trial [44]. It aimed to determine the optimal dose and tolerability of vericiguat in 456 clinically stable patients with a LVEF $<45 \%$ within 4 weeks of a WCHF event. The patients were equally randomized to receive either placebo or 1 of 4 daily doses of oral vericiguat $(1.25 \mathrm{mg}$, $2.5 \mathrm{mg}, 5 \mathrm{mg}, 10 \mathrm{mg}$ ) for 12 weeks. No significant difference between the pooled vericiguat group and placebo was reported for the primary outcome measure which was change in log-transformed level of N-terminal pro-B-type natriuretic peptide (NTproBNP) between baseline and Week 12. In parallel, HFH tended to be reduced in the 2 highest dose groups ( 5 and $10 \mathrm{mg}$ ), this effect was less visible with CV death. Some hypotension and syncopes were noted after administration of the first doses. However, after this initiation phase, vericiguat showed no effect on blood pressure (BP) although a vasodilatation and reduction of systolic BP were hypothesized given the effect of sGC stimulators such as riociguat on pulmonary hypertension [26,27]. In the vericiguat 10 
$\mathrm{mg} /$ day group, treatment-emergent syncope and hypotension were more common although this effect tended to occur within the first 2 weeks of randomization while receiving the $2.5-\mathrm{mg}$ dose.

VICTORIA was a Phase 3 global randomized, double-blind, placebo-controlled trial which evaluated the efficacy and safety of vericiguat in patients with HFrEF with recent decompensated $\mathrm{HF}$. A total of 5,050 eligible patients were randomly assigned to receive vericiguat at $10 \mathrm{mg} /$ day $(n=2,526)$ or placebo $(n=2,524)$, in addition to recommended therapy. The primary endpoint was a composite of CV death or first $\mathrm{HFH}$, and pre-specified safety event of interest were syncope and symptomatic hypotension [29]. Inclusion criteria and baseline characteristics of participants [45] are comparable with the characteristics of participants in SOCRATES-REDUCED study (patients with worsening chronic HFrEF) with the only difference that intravenous diuretics were administered within 24 hours in Phase 2 SOCRATES-REDUCED versus 3 months before randomization in Phase 3 VICTORIA. WCHF was defined as a recent HF event or 'progressively escalating symptoms and signs of HF' requiring treatment in the outpatient, emergency room, or hospital setting and is associated with a 'markedly worse prognosis' $[3,4]$.

Results of Phase 3 VICTORIA were recently published [29]. Patients who received vericiguat had a lower incidence of CV death $(n=414 ; 16.4 \%)$ or HFH $(n=691 ; 27.4 \%)$ versus those who received placebo (CV death: $n=441 ; 17.5 \%$ / HFH: $n=747 ; 29.6 \%$ ) [29]. Syncope was reported in $4 \%$ and symptomatic hypotension in $9.1 \%$ of the patients in the vericiguat group versus $3.5 \%$ and $7.9 \%$ of the patients in the placebo group, respectively, without any significant statistical difference between both treatment groups [29]. The positive data of VICTORIA trial supported the submission of the marketing authorization application to the European Medicines Agency and new drug application to the Ministry of Health, Labor and Welfare in Japan in June 2020 [46]. These regulatory submissions were made for using vericiguat in the treatment of WCHF in adults with LVEF $<45 \%$ to reduce the risk of CV death and $\mathrm{HFH}$, in combination with other HF therapies.

\section{Competitive Environment}

\subsection{Current and Future Landscape in the Treatment of HFrEF}


Emerging therapeutic options for patients with HFrEF include sacubitril/valsartan, sodium-glucose cotransporter-2 inhibitors (SGLT2i), and omecamtiv mecarbil. This section summarizes the main characteristics of each class as compared to vericiguat.

Sacubitril/valsartan, an ARNI, simultaneously inhibits neprilysin and blocks angiotensin II type-1 receptors [47]. Sacubitril/valsartan has shown a benefit in patients with HFrEF and LVEF $<40 \%$ in the PARADIGM study. In addition, PIONEER-HF and TRANSITION Phase 4 trials support its initiation during hospitalization as soon as hemodynamic parameters have been stabilized from an acute HF episode in ACEI/ARBnaïve patients $[47,48]$. Also, sacubitril/valsartan showed encouraging results in terms of a favorable safety profile and reduction of plasma NT-ProBNP levels in patients with HFrEF $[48,49]$. As previously exposed, both sacubitril/valsartan and vericiguat are increasing cGMP levels, but through two independent pathways. The titration and use of sacubitril/valsartan is hampered by its tolerance on the BP. It is thus anticipated that the combination of sacubitril/valsartan with vericiguat might be revealed difficult into the clinics, especially in patients with WCHF and low BP.

SGLT $2 i$, also called gliflozins such as empagliflozin, canagliflozin, sotagliflozin, and dapagliflozin, are used in the treatment of type 2 diabetes mellitus (T2DM). New guidelines include empagliflozin, canagliflozin, or dapagliflozin in patients with T2DM and CVD, or at high to very high CV risk, to reduce CV events [50]. SGLT2i represent also a promising therapeutic option for patients with HFrEF [51]. The DAPA-HF Phase 3 randomized trial compared effects of dapagliflozin to placebo in 4,744 patients with chronic HF and LVEF $\leq 40 \%$, with or without diabetes, and showed a significant $26 \%$ reduction of the primary endpoint (WCHF event or CV death) [52]. In parallel, the EMPEROR-Reduced Phase 3 trial investigated effects of empagliflozin on time to CV death or first HFH in 3,730 patients with chronic HFrEF (LVEF $\leq 40 \%)$ [53]. Empagliflozin reduced significantly the primary endpoint by $25 \%$ (hazard ratio of 0.75 ; $95 \%$ confidence interval: 0.65-0.86, p-value <0.0001) [53]. Lastly, sotagliflozin, a SGLT1 and SGLT2i, was shown to reduce the rate of death from CV causes and HFH (hazard ratio of 0.67; 95\% confidence interval: 0.52-0.85; $\mathrm{p}$-value <0.001) in patients with diabetes and recent worsening HF in the SOLOIST-WHF trial [54]. Overall, these clinical trials advocate for a beneficial class effect of SGLT2i in HF patients. SGLT2i stimulate natriuresis and osmotic diuresis which reduces BP and intravascular volume, and thus decreases cardiac preload and afterload [55]. Other potential mechanisms of improved 
cardiac function with SGLT2i are, but not limited to, an increased myocardial energetics, an improved myocardial autophagy, inhibition of cardiac fibrosis, and a reduction in left ventricular mass and in the activity of the calcium/calmodulindependent protein kinase II [56]. Given that SGLT2i and vericiguat will be almost simultaneously marketed, cardiologists need to be guided to choose the most appropriate therapeutic option according to the patients' profile. There is especially no evidence from clinical trials of SGLT2i and vericiguat combined together in this setting. On a pharmacological viewpoint, SGLT2i present some additional advantages over vericiguat (Table 1). An important issue relates to the tolerance profile of vericiguat on BP as HF patients are already treated with multiple therapies that lower BP. This issue might not be observed with SGLT2 $i$, which remains to be confirmed in the upcoming clinical trials. Additional benefits of SGLT2i are their kidney-protective effects and their preventive effect in the onset of diabetes.

Omecamtiv mecarbil is a selective cardiac myosin activator that can increase cardiac contractility, reverse ventricular remodeling and reduce NT-ProBNP; without affecting intracellular myocyte calcium concentrations or myocardial oxygen consumption [62]. It thus appears that the overlap in the mechanism of action between omecamtiv mecarbil and vericiguat is limited. The hypothesis on its efficacy in the treatment of HFrEF highlights a different pathway to vericiguat and is being addressed in two Phase 3 trials (GALACTIC-HF and METEORIC-HF). In GALACTIC-HF, omecamtiv mecarbil reduced significantly but weakly the primary endpoint, time to CV death or first $\mathrm{HFH}$, by $8 \%$ in patients with chronic HFrEF $[61,63]$. METEORIC-HF is evaluating its effect on exercise capacity [64] and patient recruitment is still ongoing.

\section{Expert Opinion}

cGMP deficiency has deleterious effects on the heart and contribute to the progression of HF. Different molecules, including NO donors, PDE inhibitors, and NP analogues, target the NO-sCG-cGMP pathway but have yielded conflicting results in HF patients. Among other effects, ARNIs increase the cGMP levels and significantly improve the prognosis of HF patients with LVEF $\leq 35 \%$. Vericiguat acts as a sGC stimulator thus targeting the NO-sGC-cGMP pathway by a different mechanism that complements the 
current pharmacotherapy for HFrEF.

In the VICTORIA trial, vericiguat has shown an additional statistical add-on therapy efficacy by reducing morbi-mortality in patients with WCHF. Whether potentiating CGMP signaling might be additive or synergistic with other therapeutic options for HF requires to be better delineated. Notably, as ARNIs increase CGMP levels by indirectly blocking neprilysin-mediated degradation of NP, an open question relates to the efficacy that could be achieved by combining sacubitril/valsartan with vericiguat in patients with HFrEF. Different markers indicate that the patients included in the VICTORIA trial had a higher baseline risk as compared with patients in contemporary trials testing other therapeutic options, including ARNls, and SGLT2i. A better evaluation of the severity of HF patients might be an important determinant to guide the use of vericiguat among the available therapies. Reciprocally, there is currently limited evidence that vericiguat provides a similar benefit in patients with HFpEF, as exemplified by the lack of significant changes in markers of cardiac remodeling in the SOCRATES-preserved trial [65] or physical activity scores in the VITALITY-HFPEF trial [66]. On a side note, new ESC guidelines on the management of HF are planned for 2021. The current opinion does not aim to anticipate the recommendations to avoid contraindications, in any. However, the guidelines provide a general framework and the expert opinion would allow for a more precise/refined approach on individualized decisions.

Finally, the concept of WCHF is appealing but novel in the realm of HF management which is traditionally organized between acute and chronic stable conditions. Thus, the appropriate identification of such patients might be challenging. As such, WCHF is not defined as decompensated HF, which evokes rather more severe cases who are often hospitalized. In some countries, outpatient management of decompensated patients is not the norm, which justifies the need for an adapted treatment course. From that perspective, defining the profile of patients eligible for vericiguat treatment is important. These include worsening chronic HrEF after a decompensation event (whether or not patients received gliflozins), intolerance to other therapeutic options, and specific comorbidities (e.g. renal failure).

Future directions would potentially focus on establishing HF units and providing funds to enhance specialist nursing to ensure ambulatory follow-up with primary care physicians and cardiologists. Hence, telemedicine is expected to ensure a close but 
remote follow-up of the patients, especially in terms of vericiguat titration and safety through telemonitoring.

\section{Funding:}

Content Ed Net France provided editorial and medical writing assistance for the preparation of this manuscript; this assistance was funded by Bayer Healthcare SAS.

\section{Declaration of Interest:}

JS Hulot has received research grants from the French National Research Agency (NADHeart ANR-17-CE17-0015-02, PACIFIC ANR-18-CE14-0032-01, CORRECT_LMNA ANR19-CE17-0013-02), the ERA-Net-CVD (ANR-16-ECVD-0011-03, Clarify project), Fédération Française de Cardiologie, the Fondation pour la Recherche Médicale (EQU201903007852), and the Leducq Foundation (18CVD05). JS Hulot is also a coordinating a French PIA Project (2018-PSPC-07, PACIFIC-preserved, BPI France) and a University Research Federation against heart failure (FHU2019, PREVENT Heart Failure). The AP-HP, employer of JS Hulot, has also received research grants from Bioserenity, Sanofi, Servier, and Novo Nordisk. He has also received personal fees for lectures, advisory board participation or consultancy fees from Amgen, AstraZeneca, Bayer, Bristol-Myers Squibb, Novartis, and WeHealth. JN Trochu has received grants from Akcea and Boston as well as grants and personal fees from Abbott and Novartis. They have also received personal fees from Bayer, Vifor Pharma, Resmed, Amgen, and Corvia. E Donal has received personal fees for research facilities from GE Healthcare and has received consultancy fees from Bristol-Myer Squibb, Novartis, Alnylam, and Bayer. M Galinier has received personal fees for consultancies from Amgen, Air Liquide, AstraZeneca, Bayer, Boehringer Ingelheim, Bristol-Myers Squibb, Novartis, Pfizer, Sanofi, Servier, and Vifor Pharma. They have also given lectures for Amgen, Air Liquide, Novartis, Roche Diagnostics, and Vifor Pharma. D Logeart has received personal fees for consultancies and lectures from Amgen, AstraZeneca, Bayer, Boehringer Ingelheim, and Novartis. $P$ de Groote has received personal fees for consultancies from Actelion, AstraZeneca, Amgen, Abbott, Bayer, Merck Sharp and Dohme (MSD), Boehringer Ingelheim, Novartis, Servier, and Vifor Pharma and has given lectures for Actelion, Bayer-MSD, Novartis, Servier, and Vifor Pharma. Finally, $Y$ Juillière has received personal fees for consultancies from Abbott Vascular, Air Liquide SI, Bayer, Boston 
Scientific, GlaxoSmithKline, MSD-Schering-Plough, Novartis, and Saint-Jude Medical. They have also given lectures for Abbott Vascular, Amgen, Bristol-Myers Squibb, The Medicines Company, MSD-Schering-Plough, Novartis, Roche Diagnostics, and Servier. The authors have no other relevant affiliations or financial involvement with any organization or entity with a financial interest in or financial conflict with the subject matter or materials discussed in the manuscript apart from those disclosed.

\section{Reviewer Disclosures:}

Peer reviewers on this manuscript have no relevant financial or other relationships to disclose. 


\section{References}

Papers of special note have been highlighted as either of interest $\left(^{*}\right)$ or of considerable interest $\left(^{* *}\right)$ to readers.

1. Cook C, Cole G, Asaria P, Jabbour R, Francis DP. The annual global economic burden of heart failure. Int J Cardiol. 2014;171(3):368-376. doi: 10.1016/j.ijcard.2013.12.028.

2. Savarese G, Lund LH. Global public health burden of heart failure. Card Fail Rev. 2017;3(1):7-11. doi: 10.15420/cfr.2016:25:2.

3. Butler J, Yang M, Manzi MA, et al. Clinical course of patients with worsening heart failure with reduced ejection fraction. J Am Coll Cardiol. 2019;773(8):935-944. doi: 10.1016/j.jacc.2018.11.049.

4. EMA Guideline on clinical investigation of medicinal products for the treatment of chronic heart failure, July 2017. Available at: https: //www.ema.europa.eu/en/documents/scientific-guideline/guidelineclinical-investigation-medicinal-products-treatment-chronic-heart-failurerevision-2_en.pdf Last access: 23 March 2021.

5. Juenger J, Schellberg D, Kraemer S, et al. Health related quality of life in patients with congestive heart failure: comparison with other chronic diseases and relation to functional variables. Heart. 2002;87(3):235-241. doi: 10.1136/heart.87.3.235.

6. Hoekstra T, Lesman-Leegte I, van Veldhuisen DJ, Sanderman R, Jaarsma T. Quality of life is impaired similarly in heart failure patients with preserved and reduced ejection fraction. Eur J Heart Fail. 2011;13(9):1013-1018. doi: 10.1093/eurjhf/hfr072.

7. Ponikowski P, Voors AA, Anker SD, et al.; Authors/Task Force Members; Document Reviewers. 2016 ESC Guidelines for the diagnosis and treatment of acute and chronic heart failure: The Task Force for the diagnosis and treatment of acute and chronic heart failure of the European Society of Cardiology (ESC). Developed with the special contribution of the Heart Failure Association (HFA) of the ESC. Eur $J$ Heart Fail. 2016;18(8):891-975. doi: 10.1002/ejhf.592.

** This document includes European guidelines for the diagnosis and treatment of acute and chronic heart failure.

8. Breitenstein S, Roessig L, Sandner P, Lewis KS. Novel SGC stimulators and SGC activators for the treatment of heart failure. Handb Exp Pharmacol. 2017;243:225- 
247. doi: 10.1007/164_2016_100.

9. Greene SJ, Gheorghiade M, Borlaug BA, et al. The CGMP signaling pathway as a therapeutic target in heart failure with preserved ejection fraction. J Am Heart Assoc. 2013;2(6):e000536. doi: 10.1161/JAHA.113.000536.

* Review of the cGMP signaling cascade as a therapeutic target in heart failure with preserved ejection fraction.

10. Tsai EJ, Kass DA. Cyclic GMP signaling in cardiovascular pathophysiology and therapeutics. Pharmacol Ther. 2009;122(3):216-238. doi: 10.1016/j.pharmthera.2009.02.009.

11. Sandner P, Zimmer DP, Todd Milne G, Follmann M, Hobbs A, Stasch JP. Correction to: Soluble guanylate cyclase stimulators and activators. Handbook of Experimental Pharmacology. Springer, Berlin, Heidelberg. 2019.

** A review of soluble guanylate cyclase stimulators and activators.

12. Pieske B, Butler J, Filippatos G, et al.; SOCRATES Investigators and Coordinators. Rationale and design of the SOluble guanylate Cyclase stimulatoR in heArT failurE Studies (SOCRATES). Eur J Heart Fail. 2014;16(9):1026-1038. doi: 10.1002/ejhf.135.

* Two Phase 2 randomized studies exploring the pharmacodynamic effects, safety and tolerability, and pharmacokinetics of vericiguat versus placebo.

13. Sandner P. From molecules to patients: exploring the therapeutic role of soluble guanylate cyclase stimulators. Biol Chem. 2018; 399(7): 679-690. doi: 10.1515/hsz2018-0155.

14. Vettel C, Lämmle S, Ewens S, et al. PDE2-mediated CAMP hydrolysis accelerates cardiac fibroblast to myofibroblast conversion and is antagonized by exogenous activation of CGMP signaling pathways. Am J Physiol Heart Circ Physiol. 2014;306:H1246-H1252. doi: 10.1152/ajpheart.00852.2013.

15. Fan D, Takawale A, Lee J, Kassiri Z. Cardiac fibroblasts, fibrosis and extracellular matrix remodeling in heart disease. Fibrogenesis Tissue Repair. 2012;5(1):15. doi: 10.1186/1755-1536-5-15.

16. Borbély A Falcao-Pires I, van Heerebeek L, et al. Hypophosphorylation of the Stiff N2B titin isoform raises cardiomyocyte resting tension in failing human myocardium. Circ Res. 2009;104:780-786. doi: 10.1161/CIRCRESAHA.108.193326.

17. Buys ES, Sips $P$, Vermeersch $P$, et al. Gender-specific hypertension and 
responsiveness to nitric oxide in sGCalpha1 knockout mice. Cardiovasc Res. 2008;79:179-186. doi: 10.1093/cvr/cvn068.

18. Cawley SM, Kolodziej S, Ichinose F, Brouckaert P, Buys ES, Bloch KD. sGC\{alpha\}1 mediates the negative inotropic effects of NO in cardiac myocytes independent of changes in calcium handling. Am J Physiol Heart Circ Physiol. 2011;301(1):H157H163. doi: 10.1152/ajpheart.01273.2010.

19. LeWinter MM, Granzier H. Cardiac titin: a multifunctional giant. Circulation. 2010;121(19):2137-2145. doi:10.1161/CIRCULATIONAHA.109.860171.

20. Armstrong PW, Roessig L, Patel MJ, et al. A multicenter, randomized, double-blind, placebo-controlled trial of the efficacy and safety of the oral soluble guanylate cyclase stimulator: the VICTORIA trial. JACC Heart Fail. 2018;6(2):96-104. doi: 10.1016/j.jchf.2017.08.013.

** A paper describing the objectives and methodology of VICTORIA trial, the first Phase 3 study of vericiguat, a novel SGC stimulator designed to enhance outcomes in patients with high-risk heart failure with reduced ejection fraction with progression of chronic heart failure despite optimized standard of care therapy.

21. Boettcher M, Thomas D, Mueck W, et al. Safety, pharmacodynamic, and pharmacokinetic characterization of vericiguat: results from six phase I studies in healthy subjects. Eur J Clin Pharmacol. 2021;77(4):527-537. doi: 10.1007/s00228020-03023-7.

22. Follmann M, Ackerstaff $J$, Redlich $G$, et al. Discovery of the soluble guanylate cyclase stimulator vericiguat (BAY 1021189) for the treatment of chronic heart failure. J Med Chem. 2017;60:5146-5161. doi: 10.1021/acs.jmedchem.7b00449.

23. Archer SL, Huang JM, Hampl V, Nelson DP, Shultz PJ, Weir EK. Nitric oxide and CGMP cause vasorelaxation by activation of a charybdotoxin-sensitive $K$ channel by cGMP-dependent protein kinase. Proc Natl Acad Sci. 1994;91(16):7583-7587. doi: 10.1073/pnas.91.16.7583.

24. Premer C, Kanelidis AJ, Hare JM, Schulman IH. Rethinking endothelial dysfunction as a crucial target in fighting heart failure. Mayo Clin Proc Innov Qual Outcomes. 2019;3(1):1-13. doi: 10.1016/j.mayocpiqo.2018.12.006.

25. Kolijn D, Kovács Á, Herwig $M$, et al. Enhanced cardiomyocyte function in hypertensive rats with diastolic dysfunction and human heart failure patients after 
acute treatment with soluble guanylyl cyclase (sGC) activator. Front Physiol. 2020;11:345. doi: 10.3389/fphys.2020.00345. PMID: 32523538; PMCID: PMC7261855.

26. Ghofrani HA, D'Armini AM, Grimminger F, et al.; CHEST-1 Study Group. Riociguat for the treatment of chronic thromboembolic pulmonary hypertension. $\mathrm{N}$ Engl J Med. 2013;369(4):319-329. doi: 10.1056/NEJMoa1209657.

27. Ghofrani HA, Galiè N, Grimminger F, et al.; PATENT-1 Study Group. Riociguat for the treatment of pulmonary arterial hypertension. N Engl J Med. 2013;369(4):330340. doi: 10.1056/NEJMoa1209655.

28. Stasch JP, Schlossmann J, Hocher B. Renal effects of soluble guanylate cyclase stimulators and activators: a review of the preclinical evidence. Curr Opin Pharmacol. 2015;21:95-104. doi: 10.1016/j.coph.2014.12.014.

29. Armstrong P, Pieske B, Anstrom KJ, et al., for the VICTORIA Study Group. Vericiguat in patients with heart failure and reduced ejection fraction. $N$ Engl J Med. 2020;382:1883-1893. doi: 10.1056/NEJMoa1915928.

** An important paper describing the results of VICTORIA trial, based on which the marketing authorization application to the European Medicines Agency and new drug application to the Ministry of Health, Labor and Welfare in Japan were submitted in June 2020.

30. Gheorghiade M, Marti CN, Sabbah HN, et al.; Academic Research Team in Heart Failure (ART-HF). Soluble guanylate cyclase: a potential therapeutic target for heart failure. Heart Fail Rev. 2013;18(2):123-134. doi: 10.1007/s10741-012-93231. PMID: 22622468.

31. Mann DL et al. Braunwald's Heart Disease: A Textbook of Cardiovascular Medicine. Elsevier/Saunders; 2015.

32. Boerrigter G, Lapp H, Burnett JC. Modulation of CGMP in heart failure: a new therapeutic paradigm. Handb Exp Pharmacol. 2009;(191):485-506. doi: 10.1007/978-3-540-68964-5_21. PMID: 19089342; PMCID: PMC3835600.

33. Felker G \& Mann D. Heart Failure: A Companion to Braunwald's Heart Disease. Elsevier; 2020.

34. Mathar I, Pavkovic M, Scheerer N, Hartmann E, Sandner P. The sGC stimulator vericiguat improved outcome in a rodent model of heart failure with preserved ejection fraction (HFpEF). Circulation. 2018;138:A15553. 
35. Stasch JP, Hobbs AJ. NO-independent, haem-dependent soluble guanylate cyclase stimulators. Handb Exp Pharmacol. 2009;(191):277-308. doi: 10.1007/978-3-54068964-5_13. PMID: 19089334.

36. Follmann M, Griebenow N, Hahn MG, Hartung I, Mais FJ, Mittendorf J, Schäfer M, Schirok H, Stasch JP, Stoll F, Straub A. The chemistry and biology of soluble guanylate cyclase stimulators and activators. Angew Chem Int Ed Engl. 2013;52(36):9442-9462. doi: 10.1002/anie.201302588. Epub 2013 Aug 20. PMID: 23963798.

37. Friebe A, Sandner P, Schmidtko A. cGMP: a unique 2nd messenger molecule - recent developments in CGMP research and development. Naunyn-Schmiedeberg's Arch Pharmacol. 2020;393:287-302. doi: 10.1007/s00210-019-01779-z

38. Yandrapalli S, Khan MH, Rochlani Y, Aronow WS. Sacubitril/valsartan in cardiovascular disease: evidence to date and place in therapy. Ther Adv Cardiovasc Dis. 2018;12(8):217-231. doi: 10.1177/1753944718784536. Epub 2018 Jun 19. PMID: 29921166; PMCID: PMC6041873.

39. Cerra MC, Pellegrino D. Cardiovascular CGMP-generating systems in physiological and pathological conditions. Curr Med Chem. 2007;14(7):585-599. doi: $10.2174 / 092986707780059715$

40. Kuhn M. Cardiology: a big-hearted molecule. Nature. 2015;519(7544):416-417. doi: 10.1038/nature14373.

41. Su J, Scholz PM, Weiss HR, et al. Differential effects of cGMP produced by soluble and particulate granylyl cyclase on mouse ventricular myocytes. Exp Biol Med (Maywood). 2005;230(4):242-250. doi: 10.1177/153537020523000403.

42. Gheorghiade M, Greene SJ, Filippatos G, et al.; COMPOSE Investigators and Coordinators. Cinaciguat, a soluble guanylate cyclase activator: results from the randomized, controlled, phase IIb COMPOSE programme in acute heart failure syndromes. Eur J Heart Fail. 2012;14(9):1056-1066. doi: 10.1093/eurjhf/hfs093.

43. Evgenov OV, Pacher P, Schmidt PM, et al. NO-independent stimulators and activators of soluble guanylate cyclase: discovery and therapeutic potential. Nat Rev Drug Discov. 2006;5(9):755-768. doi: 10.1038/nrd2038.

44. Gheorghiade M, Greene SJ, Butler J, et al.; SOCRATES-REDUCED Investigators and Coordinators. Effect of vericiguat, a soluble guanylate cyclase stimulator, on natriuretic peptide levels in patients with worsening chronic heart failure and 
reduced ejection fraction: The SOCRATES-REDUCED Randomized Trial. JAMA. 2015;314(21):2251-62. doi: 10.1001/jama.2015.15734.

* A Phase 2 randomized study exploring the effect on natriuretic peptide trajectory and tolerability of 12 weeks of treatment with 4 doses of oral vericiguat in clinically stable patients with worsening chronic heart failure and reduced left ventricular ejection fraction.

45. Pieske B, Patel MJ, Westerhout CM, et al.; VICTORIA Study Group. Baseline features of the VICTORIA (Vericiguat Global Study in Subjects with Heart Failure with Reduced Ejection Fraction) trial. Eur J Heart Fail. 2019;21(12):1596-1604. doi: 10.1002/ejhf.1664.

** Description of the baseline characteristics of patients included in VICTORIA trial.

46. Bayer. Investor News. Bayer submits vericiguat for marketing authorization in the EU and Japan for the treatment of chronic heart failure. 05 June 2020. Available at: https://www.investor.bayer.com/en/nc/news/investor-news/investornews/bayer-submits-vericiguat-for-marketing-authorization-in-the-eu-and-japanfor-the-treatment-of-chroni/ Last access: 23 March 2021.

47. EMA 2015. ENTRESTO. Summary of Product Characteristics. Available at: https://www.ema.europa.eu/en/documents/product-information/entresto-eparproduct-information_en.pdf Last access: 23 March 2021.

48. Velazquez EJ, Morrow DA, DeVore AD, et al.; PIONEER-HF Investigators. Angiotensin-neprilysin inhibition in acute decompensated heart failure. N Engl J Med. 2019;380(6):539-548. doi: 10.1056/NEJMoa1812851.

* Multicenter, randomized, double-blind, active-controlled trial that compares inhospital initiation of sacubitril-valsartan therapy with enalapril therapy, in patients who had been admitted for acute decompensated heart failure with reduced ejection fraction.

49. Wachter R, Senni M, Belohlavek J, et al.; TRANSITION Investigators. Initiation of sacubitril/valsartan in haemodynamically stabilised heart failure patients in hospital or early after discharge: primary results of the randomised TRANSITION study. Eur J Heart Fail. 2019;21(8):998-1007. doi: 10.1002/ejhf.1498.

* Multicenter, randomized, open-label trial that compares two treatment initiation modalities of sacubitril/valsartan in patients stabilized after acute heart failure.

50. Cosentino F, Grant PJ, Aboyans V, et al.; ESC Scientific Document Group. 2019 ESC 
Guidelines on diabetes, pre-diabetes, and cardiovascular diseases developed in collaboration with the EASD. Eur Heart J. 2020;41(2):255-323. doi: 10.1093/eurheartj/ehz486.

** This document includes European guidelines for the management and prevention of cardiovascular disease in subjects with, and at risk of developing, diabetes mellitus.

51. Lytvyn Y, Bjornstad P, Udell JA, Lovshin JA, Cherney DZI. Sodium glucose cotransporter-2 inhibition in heart failure: potential mechanisms, clinical applications, and summary of clinical trials. Circulation. 2017;136(17):1643-1658. doi: 10.1161/CIRCULATIONAHA.117.030012.

52. McMurray JJV, Solomon SD, Inzucchi SE, et al. Dapagliflozin in patients with heart failure and reduced ejection fraction. N Engl J Med. 2019;381(21):1995-2008. doi: 10.1056/NEJMoa1911303.

** Phase 3, placebo-controlled randomized trial evaluating the efficacy and safety of the SGLT2 inhibitor dapagliflozin in patients with heart failure and a reduced ejection fraction, regardless of the presence or not of diabetes.

53. Packer M, Anker SD, Butler J, et al.; EMPEROR-Reduced Trial Investigators. Cardiovascular and renal outcomes with empagliflozin in heart failure. N Engl J Med. 2020;383(15):1413-1424. doi: 10.1056/NEJMoa2022190. Epub 2020 Aug 28. PMID: 32865377.

54. Bhatt DL, Szarek M, Steg PG, et al.; SOLOIST-WHF Trial Investigators. Sotagliflozin in patients with diabetes and recent worsening heart failure. $\mathrm{N}$ Engl J Med. 2021;384(2):117-128. doi: 10.1056/NEJMoa2030183. Epub 2020 Nov 16. PMID: 33200892.

55. Grubić Rotkvić P, Cigrovski Berković M, Bulj N, Rotkvić L, Ćelap I. Sodium-glucose cotransporter 2 inhibitors' mechanisms of action in heart failure. World J Diabetes. 2020;11(7):269-279. doi:10.4239/wjd.v11.i7.269.

56. Lam CSP, Chandramouli C, Ahooja V, Verma S. SGLT-2 inhibitors in heart failure: current management, unmet needs, and therapeutic prospects. J Am Heart Assoc. 2019;8(20):e013389. doi: 10.1161/JAHA.119.013389.

57. Solomon SD, Claggett B, Packer M, et al. Efficacy of sacubitril/valsartan relative to a prior decompensation: The PARADIGM-HF Trial. JACC Heart Fail. 2016;4(10):816-822. doi: 10.1016/j.jchf.2016.05.002. Epub 2016 Jul 6. PMID: 
27395349.

58. McMurray JJ, Packer M, Desai AS, et al. Angiotensin-neprilysin inhibition versus enalapril in heart failure. $N$ Engl J Med. 2014;371:993-1004. doi: 10.1056/NEJMoa1409077

59. McMurray JJV, DeMets DL, Inzucchi SE, et al.; DAPA-HF Committees and Investigators. The dapagliflozin and prevention of adverse-outcomes in heart failure (DAPA-HF) trial: baseline characteristics. Eur J Heart Fail. 2019;21(11):1402-1411. doi: 10.1002/ejhf.1548. Epub 2019 Jul 15. PMID: 31309699.

60. Teerlink JR, Diaz R, Felker GM, et al. Baseline characteristics from the cardiovascular outcomes trial of omecamtiv mecarbil, GALACTIC-HF (global approach to lowering adverse cardiac outcomes through improving contractility in heart failure). JACC Heart Fail. 2020;75(1). doi: 10.1016/S0735-1097(20)31381-4.

61. Teerlink JR, Diaz R, Felker GM, et al.; GALACTIC-HF Investigators. Cardiac myosin activation with omecamtiv mecarbil in systolic heart failure. $N$ Engl $\mathrm{J}$ Med. 2021;384(2):105-116. doi: 10.1056/NEJMoa2025797. Epub 2020 Nov 13. PMID: 33185990.

62. Teerlink JR, Diaz R, Felker GM, et al. Omecamtiv mecarbil in chronic heart failure with reduced ejection fraction: rationale and design of GALACTIC-HF. JACC Heart Fail. 2020;8(4). pii: S2213-1779(20)30002-0. doi: 10.1016/j.jchf.2019.12.001.

* A paper describing the rationale and design of the GALACTIC-HF trial which is testing the hypothesis that omecamtiv mecarbil can safely improve clinical outcomes in patients with heart failure with reduced ejection fraction.

63. Clinicaltrials.gov. Registrational study with omecamtiv mecarbil/amg 423 to treat chronic heart failure with reduced ejection fraction (GALACTIC-HF). Available at: https: //clinicaltrials.gov/ct2/show/NCT02929329 Last access: 23 March 2021.

64. Clinicaltrials.gov. Study to assess the effect of omecamtiv mecarbil on exercise capacity in subjects with heart failure (METEORIC-HF). Available at: https://clinicaltrials.gov/ct2/show/NCT03759392 Last access: 23 March 2021.

65. Pieske B, Maggioni AP, Lam CSP, et al. Vericiguat in patients with worsening chronic heart failure and preserved ejection fraction: results of the SOluble guanylate Cyclase stimulatoR in heArT failurE patientS with PRESERVED EF (SOCRATESPRESERVED) study. Eur Heart J. 2017;38(15):1119-1127. doi: 
10.1093/eurheartj/ehw593.

66. Armstrong PW, Lam CSP, Anstrom KJ, et al; VITALITY-HFpEF Study Group. Effect of vericiguat vs placebo on quality of life in patients with heart failure and preserved ejection fraction: the VITALITY-HFpEF randomized clinical trial. JAMA. 2020;324(15):1512-1521. doi: 10.1001/jama.2020.15922. Erratum in: JAMA. 2021;325(5):494. 


\section{Figure Legends}

A.

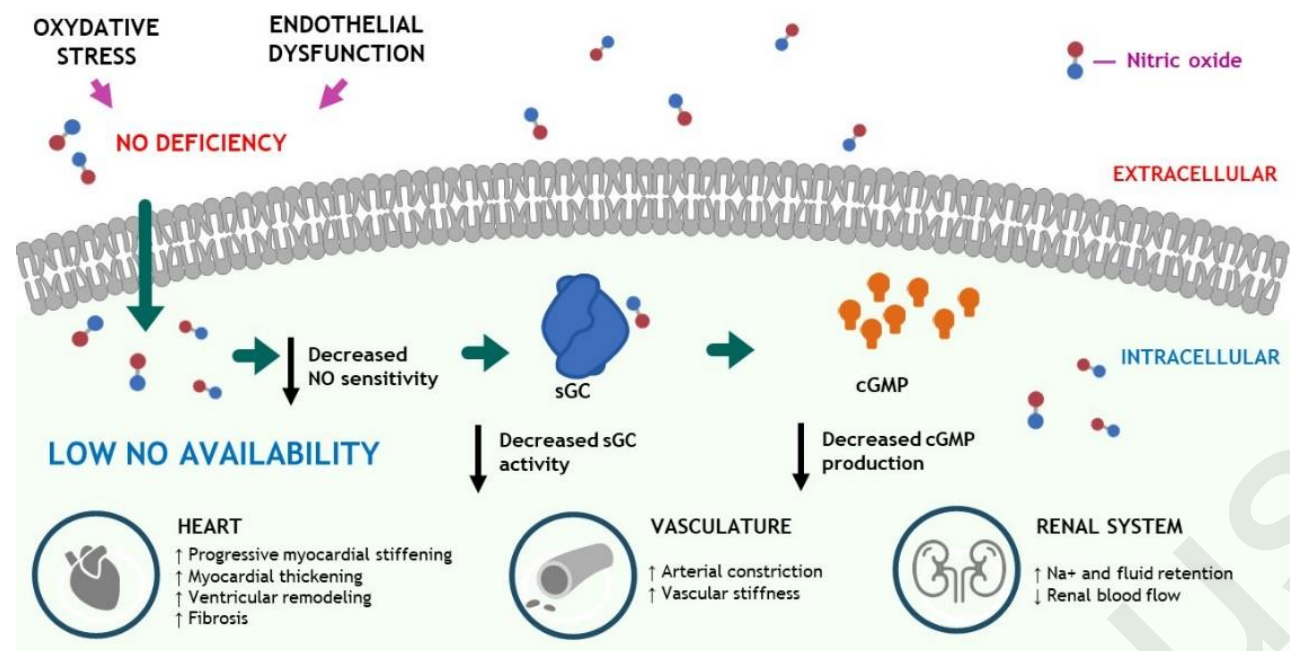

B.

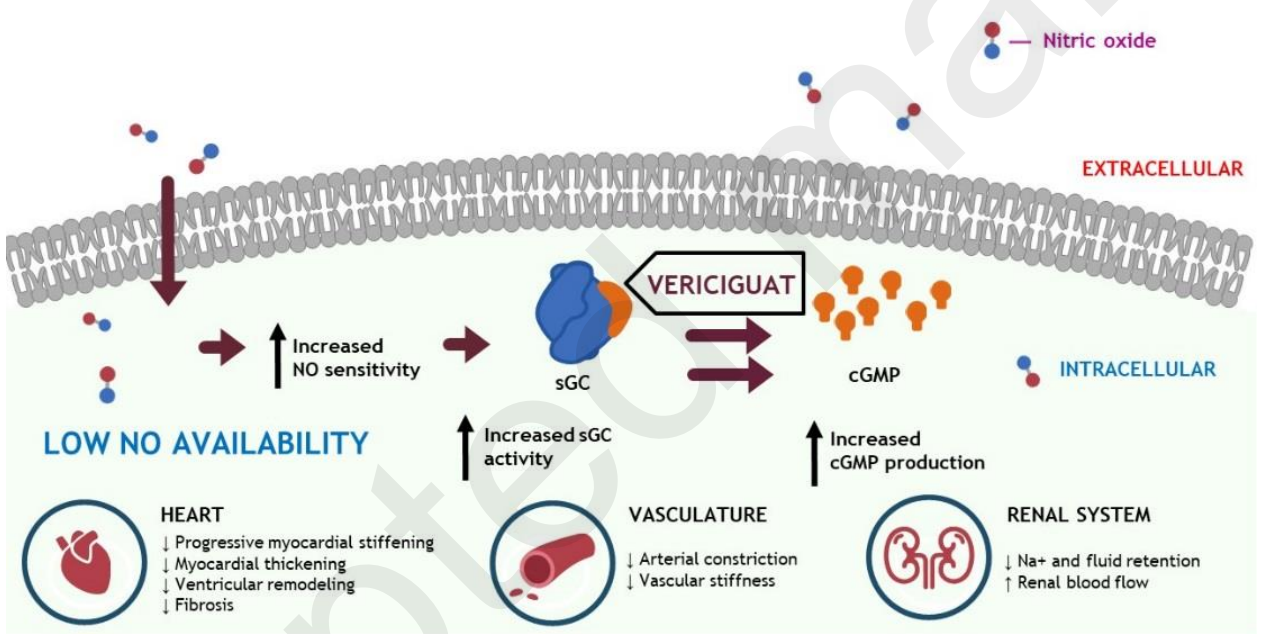

Figure 1. Mechanism of action of vericiguat and related clinical effects $[8,20,22,30$ -

34].

A: The NO-sGC-cGMP pathway is not currently medically addressed. Under normal conditions, NO travels through the cell membrane and binds to SGC which in turn leads to an increased availability of cGMP. However, under conditions of oxidative stress and endothelial dysfunction commonly found in patients with HF, there is a significant deficiency in NO. This low NO availability leads to a decreased activity of the $S G C$ as well as a reduction in its sensitivity to NO. Ultimately, this results in a reduction in cGMP production that has deleterious downstream effects for the heart, vasculature and renal system. B: Vericiguat helps restore the deficient NO-sGC-cGMP pathway in patients with HF. By directly stimulating the $\mathrm{SGC}$ independently, it helps increase its activity independent of endogenous NO, which results in an increased production of CGMP. This in turn leads to a reduction of the deleterious effects of the deficient NO-sGC-cGMP pathway on the heart, vasculature and renal system with a reduction in myocardial and 
vascular stiffening, a reduction in fibrosis of the myocardium etc. Also, by increasing sGC sensitivity to NO, its CGMP output is further improved.

Abbreviations: cGMP: cyclic guanosine monophosphate; HF: heart failure; Na: sodium; NO: nitric oxide; sGC: soluble guanylate cyclase. 


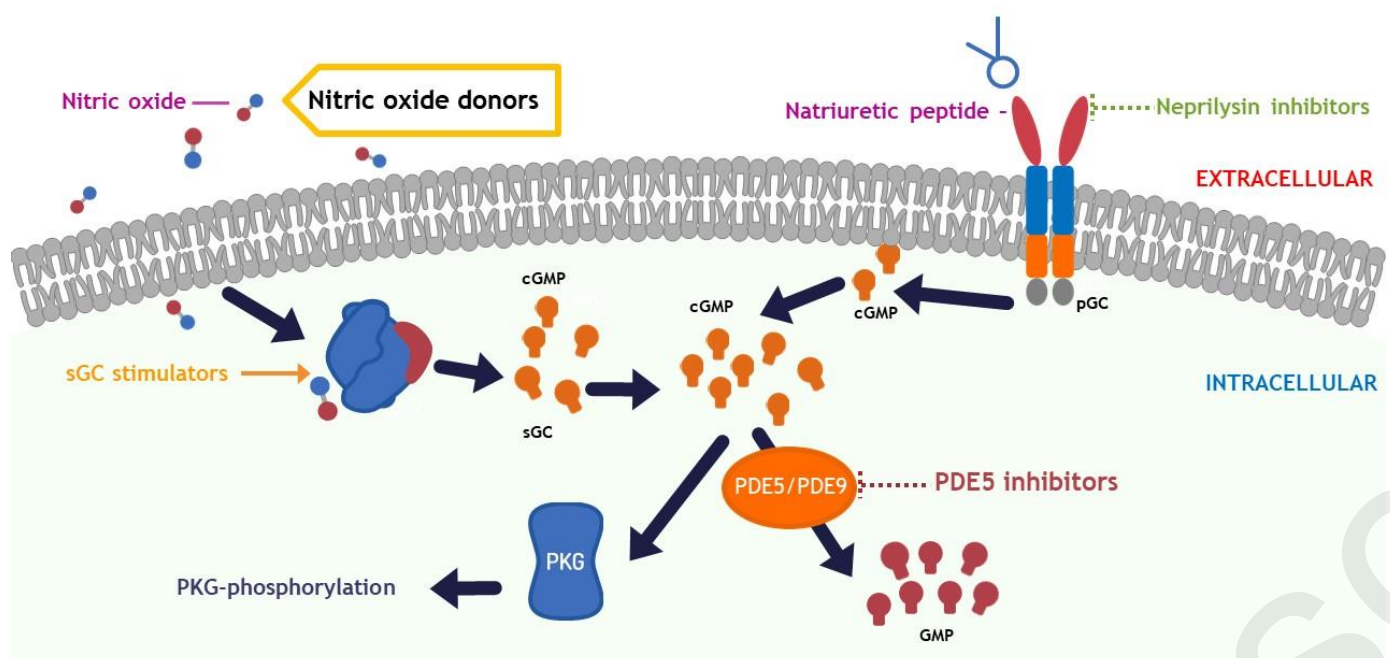

Figure 2. CGMP augmentation through different pathways in the treatment of heart failure with vericiguat (NO-sGC-cGMP pathway) and sacubitril/valsartan described as ARNI (NP-pGC-cGMP pathway) $[8,9,13,35,39]$.

This graph shows the synergistic effect of $S G C$ stimulators and neprilysin inhibitors (e.g. ARNI) in augmenting the cGMP availability via the NO-sGC-cGMP and the particulate CG-cGMP pathways respectively.

Abbreviations: ARNI: angiotensin receptor-neprilysin inhibitor; cGMP: cyclic guanosine monophosphate; GTP: guanosine triphosphate; NO: nitric oxide; NP: natriuretic peptides; pCG: particulate guanylate cyclase; PDE: phosphodiesterase; PKG: protein kinase G; sGC: soluble guanylate cyclase. 
Table 1. Comparison of data between Phase 3 HFrEF studies.

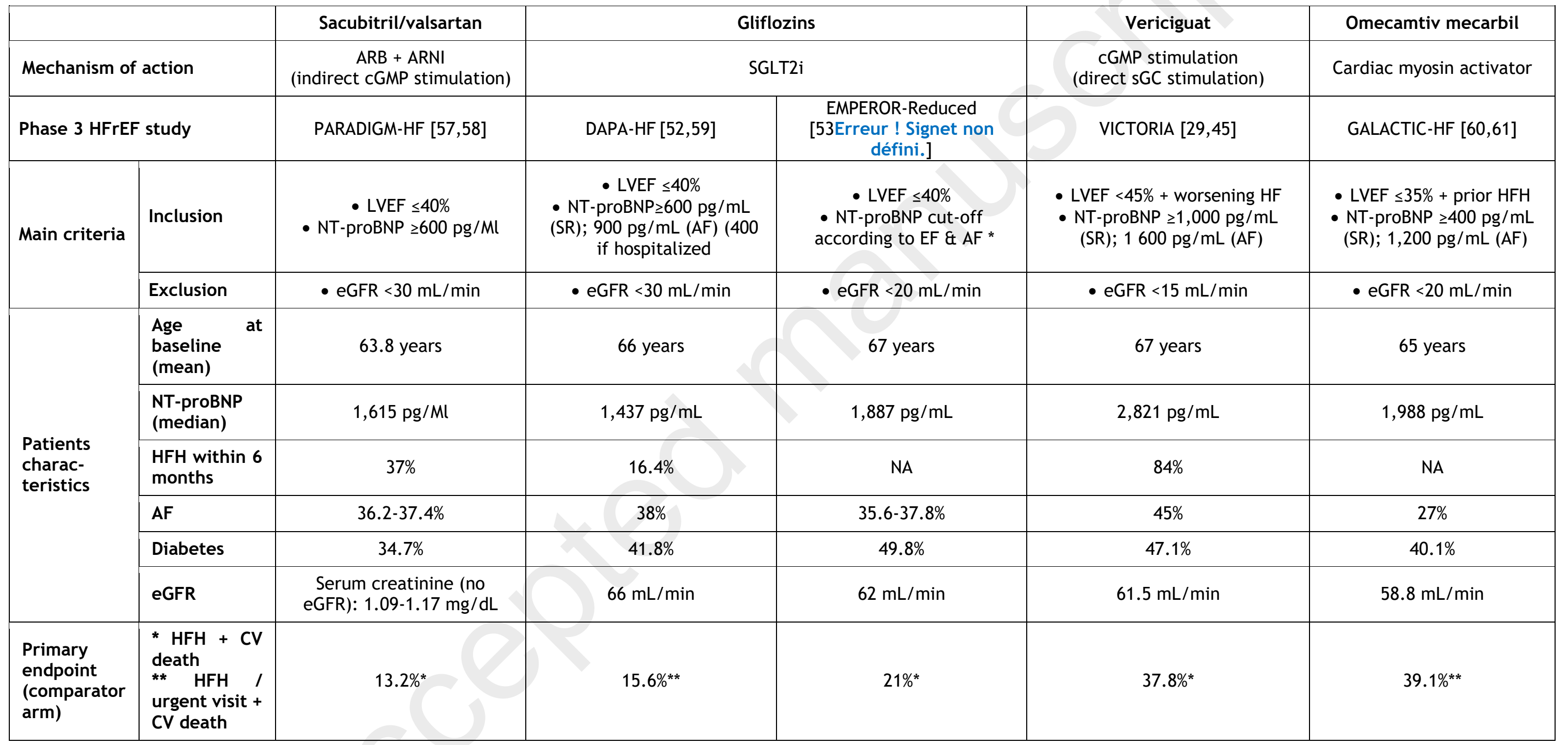




\begin{tabular}{|c|c|c|c|c|c|}
\hline & Sacubitril/valsartan & \multicolumn{2}{|c|}{ Gliflozins } & Vericiguat & Omecamtiv mecarbil \\
\hline $\begin{array}{l}\text { Median follow-up time } \\
\text { (months) }\end{array}$ & 27 & 18.2 & 16 & 10.8 & 21.8 \\
\hline Prescription information & $\begin{array}{l}\text { Bid; titration } \\
\text { (mean dose: } 375 \mathrm{mg} \\
\text { in Paradigm-HF) }\end{array}$ & \multicolumn{2}{|c|}{ Od; no titration } & $\begin{array}{c}\text { Od; titration } \\
\text { (90.3\% at target dose in } \\
\text { VICTORIA) }\end{array}$ & Bid; titration \\
\hline Safety profile & $\begin{array}{l}\text { Higher proportions of } \\
\text { patients with hypotension } \\
\text { and non-serious } \\
\text { angioedema versus the } \\
\text { enalapril group }\end{array}$ & $\begin{array}{c}\text { No difference in the } \\
\text { frequency of adverse } \\
\text { events related to volume } \\
\text { depletion, renal } \\
\text { dysfunction, and } \\
\text { hypoglycemia between } \\
\text { treatment groups (versus } \\
\text { placebo) }\end{array}$ & $\begin{array}{l}\text { - Slower annual rate of } \\
\text { decline in the eGFR with } \\
\text { empagliflozin versus } \\
\text { placebo } \\
\text { - Lower risk of serious } \\
\text { renal outcomes with } \\
\text { empagliflozin } \\
\text { - More frequent } \\
\text { uncomplicated genital } \\
\text { tract infection with } \\
\text { empagliflozin }\end{array}$ & $\begin{array}{l}\text { - Symptomatic hypotension: } \\
\text { 9.1\% in the vericiguat group } \\
\text { versus } 7.9 \% \text { in the placebo } \\
\text { group ( } p \text {-value-0.12) } \\
\text { - Syncope: } 4 \% \text { in the vericiguat } \\
\text { group versus } 3.5 \% \text { in the } \\
\text { placebo group } \\
\text { - More frequent anemia in the } \\
\text { vericiguat group }(7.6 \%) \text { versus } \\
\text { the placebo group }(5.7 \%)\end{array}$ & $\begin{array}{l}\text { - No difference in } \\
\text { the frequency of } \\
\text { adverse events } \\
\text { related to cardiac } \\
\text { ischemic events } \\
\text { - Similar rates of } \\
\text { arrhythmic events }\end{array}$ \\
\hline What to monitor & & & & rolytes & \\
\hline
\end{tabular}

${ }^{*}$ NT-proBNP $\geq 1,000 \mathrm{pg} / \mathrm{mL}$ for EF $31-35 \% ; \geq 2,500 \mathrm{pg} / \mathrm{mL}$ for EF $36-40 \%, \geq 600 \mathrm{pg} / \mathrm{mL}$ for EF $<30 \%$. These NT-proBNP thresholds were doubled in patients with AF.

Abbreviations: AF: atrial fibrillation; ARB: angiotensin receptor blocker; ARNI: receptor-neprilysin inhibitor; Bid: bis in die (twice a day); cGMP: cyclic guanosine monophosphate; CV: cardiovascular; EF: ejection fraction; eGFR: estimated glomerular filtration rate; HFH: heart failure hospitalization; HFrEF: heart failure with reduced ejection fraction; LVEF: left ventricular ejection fraction; NA: not applicable; NT-proBNP: N-terminal pro-B-type natriuretic peptide; OD: omne in die (once a day); sGC: soluble guanylate cyclase; SGLT2i: sodiumglucose cotransporter-2 inhibitor; SR: sinus rhythm. 